\title{
Efectos de la suplementación con aceite de pescado sobre la concentración de ácidos grasos en carne de cerdo
}

\author{
Pochon, D.O. ${ }^{1}(\dagger)$; Judis, M.A. ${ }^{2}$; Koslowski, H.A. ${ }^{1,3}$; Picot, J.A.'; Navamuel, J.M. ${ }^{1}$ \\ ${ }^{1}$ Cátedra de Bioestadística, ${ }^{3}$ Cátedra de Nutrición y Alimentación Animal, Facultad de Ciencias Veterinarias, \\ UNNE, Sargento Cabral 2139, Corrientes (3400), Argentina. Tel. 03794-425753. ${ }^{2}$ Facultad de Agroindustrias, \\ Universidad Nacional del Chaco Austral, Sáenz Peña (Provincia de Chaco, Argentina). \\ E-mail: japicot@gmail.com.
}

\begin{abstract}
Resumen
Pochon, D.O.; Judis, M.A.; Koslowski, H.A.; Picot, J.A.; Navamuel, J.M.: Efectos de la suplementación con aceite de pescado sobre la concentración de ácidos grasos en carne de cerdo. Rev. vet. 23: 2, 120-125, 2012. Las grasas son excelentes fuentes de energía para ser adicionadas a la alimentación de cerdos. El objetivo del trabajo fue analizar los niveles de ácidos grasos en carne de cerdos alimentados con harina integral de mandioca, maíz molido y expeller de soja, suplementados con aceite de pescado. Se utilizaron 4 cerdos, con tres repeticiones por tratamiento, bajo un diseño completamente aleatorizado, durante 21 días (7 días de adaptación y 14 días de medición). Se ensayaron 4 dietas: T1: dieta base (DB) preparada con maíz molido (66\%) más expeller de soja (31\%); T2: DB + el agregado de 5\% de aceite de pescado a la ración; T3: DB a la cual se le reemplazó el $40 \%$ de maíz por mandioca integral (DBM); y $\mathrm{T} 4=\mathrm{DBM}+$ el agregado de $5 \%$ de aceite de pescado a la ración. Se efectuaron estadísticas descriptivas y análisis de la varianza a una sola vía, rechazando la hipótesis nula con un nivel de riesgo $\alpha$ del 5\%. Las medias se compararon mediante la prueba de Tukey. En ningún tratamiento los promedios registraron diferencias significativas para los ácidos grasos saturados, mono-insaturados ni poli-insaturados. Se concluye que la inclusión de aceite de pescado en la dieta no produce modificaciones en el perfil de ácidos grasos de la carne de cerdo.
\end{abstract}

Palabras clave: cerdo, suplementación, aceite de pescado, ácidos grasos en carne.

\begin{abstract}
Pochon, D.O.; Judis, M.A.; Koslowski, H.A.; Picot, J.A.; Navamuel, J.M.: Effects of fish oil supplementation in pig meat fatty acids concentration. Rev. vet. 23: 2, 120-125, 2012. Fats are excellent sources of energy for pig feeding. The objective of this work was to analyze the levels of fatty acids in meat of pigs fed with diets including cassava flour, corn ground, and expeller soybean, supplemented with fish oil. Four pigs were used in three repetitions per treatment. The assay was carried out using a completely randomized design during 21 days ( 7 days for adaptation and 14 days for determinations). The experience included 4 diets; T1: basal diet (DB) prepared with ground corn (66\%) plus soybean expeller (31\%), T2: DB + fish oil 5\% (by weight), T3: DB with $40 \%$ corn replaced by integral cassava (DBM) and T4 $=\mathrm{DBM}+$ fish oil $5 \%$ (by weight). Descriptive statistics and analysis of variance were performed by one-way, where the null hypothesis was rejected with an $\alpha$ risk level of $5 \%$. Means were compared using the Tukey's test. Treatment averages did not show significant differences for saturated, mono-unsaturated nor poly-unsaturated fatty acids. It can be concluded that fish oil in pig diets does not cause changes in fatty acid meat profile.
\end{abstract}

Key words: pig, fish oil supplementation, fatty acids in meat.

\section{INTRODUCCIÓN}

La producción mundial de carne de cerdo aumentó a partir del año 2004 a poco más de 100 millones de toneladas, como causa de la situación sanitaria bovina y el alza de los precios de la carne de aves. Esta si-

Proyecto SGCYT-UNNE B045/2006

Recibido: 5 julio 2012 / Aceptado: 28 septiembre 2012 tuación alentó un crecimiento del $4 \%$ de la producción porcina en los países en desarrollo, particularmente en Asia, donde los precios subieron por la escasez de otros tipos de carne ${ }^{8}$. Nuestro país presenta una marcada reducción del stock de ganado bovino, con un consumo humano nacional de 54,4 kg/habitante/año, lo cual implica una merma de $22,1 \%$ desde el año 2009 al primer semestre de 2011, situación que favorece aún más el reemplazo por el consumo de otros tipos de proteínas. 
El volumen de producción y consumo mundial de carne de cerdo es el más importante entre las restantes carnes, registrando en los últimos 6 años una proporción de $44 \%$ para el cerdo, $29 \%$ para las aves y $24 \%$ para la carne vacuna. Los países del MERCOSUR, con excepción de Brasil, son deficitarios en carne porcina pero tienen un gran potencial de producción basado en la producción competitiva de granos y mano de obra más barata. Brasil es el único exportador de la región pero tiene un bajo consumo per cápita $(9,4 \mathrm{~kg} / \mathrm{habitan}-$ te/año) que en caso de aumentar puede comprometer su saldo exportable ${ }^{14}$. En nuestro país se observa un incremento que llega a 9,5 kg/habitante/año en el consumo de carne fresca y manufacturada (chacinados).

Luego de un prolongado tiempo negativo para la producción tanto regional como nacional, hoy se observa un creciente y paulatino aumento de la composición del stock de los planteles, lo que se traduce en forma importante en la faena, acusando una rentabilidad positiva para los criadores eficientes. Dicha producción si bien hoy es complementaria, requiere urgente tecnificación y se inserta como un recurso alternativo de producción, cuyas exigencias tienen que ser atendidos para optimizar los índices productivos y elevar la calidad carnicera del producto final satisfaciendo de ese modo las necesidades de un consumidor cada vez más exigente ${ }^{2}$.

En la alimentación porcina los ingredientes de la dieta compiten cada vez más con la alimentación humana ${ }^{14,18}$. Una alternativa para promover la sustentabilidad del sistema es el uso de recursos locales de alta producción de biomasa y energía renovable, con bajos requerimientos de insumos y aplicación de tecnología simple en la elaboración de las dietas, sustituyendo total o parcialmente las materias primas de origen foráneo ${ }^{1}$.

Las raciones basadas en el maíz y la harina de soja siguen siendo los estándares en la alimentación para cerdos, por lo que su crianza está prácticamente circunscripta a las regiones donde se producen estos insumos básicos. Tal situación crea la necesidad de buscar fuentes alternativas, tanto proteicas como energéticas que resulten nutricionalmente aceptables. En Argentina dichas zonas están bien delimitadas y el gran volumen de la producción de ambos cultivos coincide con las áreas de mayor producción de cerdos.

En un programa de alimentación de cerdos existe una gran variedad de ingredientes que pueden utilizarse en la formulación de una dieta. Su nivel de inclusión estará determinado por la composición nutricional, las restricciones nutricionales para diferentes etapas productivas y el requerimiento de nutrimentos que se quiera satisfacer. Las fuentes de ingredientes para la elaboración de raciones o balanceados comerciales pueden dividirse en cuatro categorías: energía, proteínas, vitaminas y minerales.

Lípidos y carbohidratos constituyen el componente energético de las dietas. El contenido de energía juega un papel determinante en el comportamiento productivo de monogástricos (aves y cerdos) y constituye el componente individual más costoso de la ración. El gasto en alimentación representa el $60-70 \%$ del costo total de producción de carne de cerdo, siendo por tanto el factor fundamental que incide sobre la rentabilidad. No sólo existe el ya mencionado problema de la competencia alimentaria entre porcinos y humanos, sino también el hecho de que la región no posee las condiciones climáticas ni el avance tecnológico que permitan obtener cosechas competitivas de los cultivos.

La fuente de energía de la ración constituye el principal costo $(42 \%)$ en la alimentación del cerdo. La existencia y los precios de los cereales adecuados, son factores decisivos para lograr el éxito de una explotación porcina en mercados altamente competitivos, de allí que la producción de cerdos esté relacionada con la utilización de alta tecnología y el uso de cereales. Ello genera dependencia foránea, lo cual torna insostenible al sistema, por lo cual son importantes las alternativas alimenticias ${ }^{9}$.

En regiones tropicales y subtropicales existen innumerables fuentes que podrían emplearse para asegurar sistemas de producción sostenibles y a su vez contribuir a la descontaminación ambiental ${ }^{3}$. Sin embargo, la información sobre el valor nutritivo de estos alimentos es muy escasa y para incluirlos en las dietas resulta necesario caracterizar su fracción fibrosa. Esta incluye no sólo su composición química, sino las propiedades físicas que posibilitan determinar su calidad, a la vez que influyen en los procesos digestivos y fisiológicos del animal ${ }^{18}$ y por ende en su respuesta productiva.

En el cerdo, las grasas constituyen excelentes fuentes de energía, especialmente para ser utilizadas en los períodos de altas temperaturas ambientales como compensación de la disminución del consumo de ración. Los tipos más usado son grasas y aceites animales (pollo, bovino, pescado, cerdo) y los aceites vegetales (de soja, soja integral tostada o extrusada, coco y otros) ${ }^{4}$. El hecho de reducir el consumo de ración, no significa que deba proporcionalmente elevarse el nivel de todos los nutrientes, sino proporcionar ingredientes más digeribles y que generen menos calor metabólico durante la digestión. Así, agregar grasa o aceite no significa aumentar la energía metabolizable de la ración, sino elevar la proporción de energía proveniente de esas fuentes energéticas. Por ser más digeribles que el almidón y generar menos calor metabólico en el proceso de la digestión, es conveniente sustituir parte del mismo, en climas con altas temperaturas ambientales ${ }^{18}$. Asimismo, la inclusión de aceites vegetales como fuentes de ácidos grasos $\omega-3$ y $\omega-6$ permiten obtener productos cárnicos (lomo, pierna) con grasas más sanas para la alimentación humana ${ }^{19}$. La principal función de los triglicéridos es el almacenamiento de energía ${ }^{10}$. Los ácidos grasos de los triglicéridos utilizados en la alimentación de cerdos tienen 14, 16 o 18 átomos de carbono; ácidos grasos con longitudes superiores (22 a 26 átomos de carbono) son encontradas en los aceites de pescado.

El cerdo tiene una limitada capacidad para insertar dobles enlaces en las cadenas carbonadas (desatura- 
ción). Ellos no son capaces de producir desaturaciones entre el último doble enlace y el grupo metilo terminal, por lo que la longitud de la cadena en este segmento es fija y define la familia de ácidos grasos. Así, el ácido oleico, se conoce como $\omega-9$, el palmitoleico como $\omega-7$, el linoleico como $\omega-6$ y el linolénico como $\omega-3$. Los ácidos $\omega-6$ y $\omega-3$ son metabolizados in vivo a moléculas de mayor peso molecular, tales como prostaglandinas, prostaciclinas y tromboxanos, las cuales tienen particular importancia como reguladores de la función celular. Debido a que los ácidos linoleico y linolénico no pueden ser sintetizados a partir de otras familias, ellos son definidos como ácidos grasos esenciales ${ }^{13,20}$.

En los aceites de soja, girasol y maíz predomina el ácido linoleico y en el aceite de palma el ácido palmítico, en tanto que en la composición de ácidos grasos de los triglicéridos del cerdo predominan los ácidos palmítico y oleico, con pequeñas cantidades de ácidos esteárico y linoleico ${ }^{21}$.

En general, las investigaciones indican que la adición de 1 a $5 \%$ de grasa en dietas para cerdos en crecimiento y engorde mejora la conversión alimenticia y la ganancia de peso diaria, sin afectar la calidad de la canal ${ }^{5-7,21}$. Sin embargo, la adición de grasa en dietas para cerdos finalmente dependerá de los costos. Dicha adición usualmente incrementa el costo de la dieta, por

Tabla 1. Composición de los ingredientes utilizados en cada dieta (\%).

\begin{tabular}{lcccc}
\hline ingrediente & $\mathrm{T} 1$ & $\mathrm{~T} 2$ & $\mathrm{~T} 3$ & $\mathrm{~T} 4$ \\
& (DB) & (DBA) & (DBM) & (DBMA) \\
\hline maíz molido & 66,2 & 66,2 & 39,72 & 39,72 \\
mandioca integral & 0 & 0 & 26,48 & 26,48 \\
expeller de soja & 31,3 & 31,3 & 31,3 & 31,3 \\
núcleo & 2,5 & 2,5 & 2,5 & 2,5 \\
aceite de pescado* & - & 5 & - & 5 \\
total & 100 & 100 & 100 & 100 \\
\hline
\end{tabular}

T1 a T4: tratamientos. DB: dieta base, DBA: dieta base + aceite de pescado, DBM: dieta base reemplazando maíz por mandioca, DBMA: la anterior + aceite de pescado. El núcleo contenía minerales y aminoácidos. *Adicionado a posteriori.

Tabla 2. Composición del aceite de pescado adicionado.

\begin{tabular}{lc}
\hline ácido graso & $(\%)$ \\
\hline gamma linoleico $18: 3 \omega 3$ & 1,10 \\
estearidónico $18: 4 \omega 3$ & 2,58 \\
eicosapentaenoico $20: 3 \omega 3 \mathrm{n} 6$ & 0,16 \\
araquidónico 20:4 $\omega 3$ & 0,62 \\
eicosapentaenoico 20:5 $\omega 3 \mathrm{EPA}$ & 7,18 \\
heneicosapentaenoico 21:5 $\omega 3$ & 0,23 \\
docosapentanoico 22:5 $\omega 3 \mathrm{DPA}$ & 0,70 \\
docosahexaenoico 22:6 $\omega 3 \mathrm{DHA}$ & 16,47 \\
ácidos grasos n-3 & 29,04 \\
ácidos grasos n-6 & 3,52 \\
ácidos grasos poliinsaturados (PUFA) & 32,56 \\
ácidos grasos libres & 0,43 \\
otros & 5,41 \\
\hline
\end{tabular}

lo cual deberá ser compensada por un incremento productivo ${ }^{17}$. La grasa adicionada a la dieta de cerdos debe estar balanceada para mantener constantes las proporciones de energía y otros nutrientes críticos como las proteínas. Así, adicionar grasa sin ajustar la proteína, puede causar que los cerdos coman menos con sólo una leve mejoría en la eficiencia. No obstante, adicionando grasa e incrementando la proteína, usualmente se generarán ganancias más rápidas y mejoras en la conversión alimenticia, cuando se compara con cerdos alimentados con dietas sin la adición de grasa.

El objetivo del trabajo fue analizar los niveles de ácidos grasos registrados en carne de cerdos alimentados con dietas conteniendo harina integral de mandioca y suplementados con aceite de pescado.

\section{MATERIAL Y MÉTODOS}

El ensayo se realizó en el módulo experimental para cerdos de la Facultad de Ciencias Veterinarias de la UNNE (Corrientes, Argentina). Se utilizaron animales cruza Landrace x Yorkshire, de similares características fenotípicas, clínicamente sanos, vacunados y desparasitados acorde al manejo sanitario habitual de la zona, respetando las normas de ética y bioseguridad.

Utilizando ingredientes como maíz molido, harina integral de mandioca, expeller de soja y aceite de pescado, se formularon cuatro dietas (tratamientos $\mathrm{T} 1$ a T4). La Tabla 1 indica la composición de cada tratamiento: T1: dieta base (DB) preparada con maíz molido (66\%) más expeller de soja (31\%), T2: DB + el agregado

Tabla 3. Nomenclatura de ácidos grasos aislados de la carne de cerdos.

\begin{tabular}{lc}
\hline ácidos grasos saturados & abreviatura \\
\hline (14:0) ácido tetradecanoico (miristico) & $(14: 0)$ \\
$(15: 0)$ ácido pentadecanoico & $(15: 0)$ \\
$(16: 0)$ ácido hexadecanoico (palmítico) & $(16: 0)$ \\
$(17: 0)$ ácido heptadecanoico & $(17: 0)$ \\
$(18: 0)$ ácido octadecanoico (esteárico) & $(18: 0)$ \\
$(20: 0)$ ácido eicosanoico (araquídico) & $(20: 0)$ \\
\hline ácidos grasos mono-insaturados & \\
\hline$(16: 1)$ cis-9 hexadecenoico (palmitoleico) & $16: 1(9)$ \\
$(17: 1)$ cis-10 heptadecenoico & $17: 1(10)$ \\
$(18: 1)$ trans-11octodecenoico (transvacénico) & trans $18: 1(11)$ \\
$(18: 1)$ cis-9 octadecenoico (oleico) & $18: 1(9)$ \\
$(24: 1)$ tetracosaenoico (nervónico) & $24: 1(15)$ \\
\hline ácidos grasos poli-insaturados & $18: 2(9,12)$ \\
\hline$(18: 2)$ cis linoleico, $\omega 6$ & $18: 3(\mathrm{n}-3)$ \\
$(18: 3)$ gamma-linoleico, $\omega$ & $18: 2(9 \mathrm{c}, 11 \mathrm{t})$ \\
$(18: 2) 9$ cis,11 trans, CLA rumenico & $18: 2(10,12)$ \\
$(18: 2)$ cis 10, cis 12, CLA linoleico & $20: 2(11,14)$ \\
$(20: 2)$ cis 11,14 eicosadienoico $\omega 6$ & $20: 3(8,11,14)$ \\
$(20: 3)$ cis $8,11,14$ eicosatrienoico $\omega 6$ & $20: 3(11,14,17)$ \\
$(20: 3)$ cis $11,14,17$ eicosatrienoico $\omega 3$ & $22: 1(9)$ \\
$(22: 1) 9-$-icosaenoico $\omega 3$ & $22: 2(\mathrm{n}-6)$ \\
$(22: 2)$ cis 13,16 docosadienoico $\omega 3$ & $22: 6(\mathrm{n}-3)$ \\
\hline &
\end{tabular}


de 5\% (de la ración) de aceite de pescado, T3: DB a la cual se le reemplazó el $40 \%$ de maíz por mandioca integral (DBM) y T4 $=$ DBM + el agregado de $5 \%($ de su peso) de aceite de pescado. Cada tratamiento incluyó un $2,5 \%$ de una mezcla (núcleo) rica en minerales y aminoácidos como lisina (principal limitante en todas las dietas para porcinos) y metionina (de bajo contenido en la raíz de mandioca).

Las cuatro dietas fueron isoenergéticas e isoproteicas. Para satisfacer las necesidades nutritivas de los animales, al momento de formular las raciones se adoptaron las recomendaciones del National Research Council (NRC) ${ }^{15}$, tomando como base las estipulaciones del Nutrient Requirements of Swine ${ }^{16}$. La composición de ácidos grasos del aceite de pescado (de mar) adicionado, se describe en la Tabla 2.

Se utilizaron 4 cerdos alojados en jaulas individuales de $3,75 \mathrm{~m}^{2}$, provistos de bebederos automáticos, efectuándose tres repeticiones por tratamiento. $\mathrm{La}$ prueba se desarrolló durante 21 días (7 días de adaptación y 14 días de medición). Luego de la faena se colectaron muestras de carne (jamón, lomo y paleta), que fueron congeladas a $-20^{\circ} \mathrm{C}$, hasta su llegada al laboratorio. Los ácidos grasos fueron extraídos de las muestras de carne aplicando la técnica de Folch. La composición de ácidos grasos de la carne de cerdo y del aceite de pescado se obtuvo en un cromatógrafo gaseoso Agilent ${ }^{\circledR}$ equipado con una columna capilar de $60 \mathrm{~m}$ de largo y $0,25 \mathrm{~mm}$ de diámetro interno (Supelco 2340) y un detector de ionización de llama. El método de cromatografía gaseosa utilizado (GC-FID) se adecuó a la norma ISO 15304. La nomenclatura utilizada para designar los ácidos grasos (acorde a las normas IUPAC - International Union of Pure and Applied Chemistry), se describen en la Tabla 3.

El ensayo se llevó a cabo utilizando un diseño completamente aleatorizado. La estadística descriptiva incluyó la determinación del promedio y error estándar. Una vez comprobados los supuestos de normalidad (Wilk-Shaphiro) y homocedasticidad de las variancias (Levene), se efectuó análisis de varianza a una vía, rechazándose la hipótesis nula con un nivel de riesgo $\alpha$ del $5 \%$. Las medias se compararon mediante la prueba de Tukey. $(\mathrm{mg} / \mathrm{g})$. cerdos (\%). $(\mathrm{mg} / \mathrm{g})$.

\section{RESULTADOS Y DISCUSIÓN}

Los perfiles de ácidos grasos de la carne (pool de jamón, lomo y paleta) de los cerdos alimentados con las dietas T1 a T4 preparadas con mandioca integral, maíz

Tabla 4. Valores obtenidos para los ácidos grasos en carne de cerdos

\begin{tabular}{lcccc}
\hline ácidos grasos & $\mathrm{T} 1$ & $\mathrm{~T} 2$ & $\mathrm{~T} 3$ & $\mathrm{~T} 4$ \\
\hline saturados & $73,20(11,33)$ & $67,12(6,01)$ & $63,6(5,57)$ & $71,15(7,30)$ \\
mono-insaturados & $66,39(5,00)$ & $74,75(7,44)$ & $64,23(7,19)$ & $80,59(9,14)$ \\
poli-insaturados & $46,47(9,09)$ & $53,07(5,13)$ & $37,86(5,47)$ & $49,76(5,68)$ \\
\hline
\end{tabular}

Valores expresados en promedio y (entre paréntesis) error estándar.

No se registraron diferencias significativas entre tratamientos.

Tabla 5. Concentraciones de ácidos grasos registradas en carne de

\begin{tabular}{lcccc}
\hline ácidos grasos & $\mathrm{T} 1$ & $\mathrm{~T} 2$ & $\mathrm{~T} 3$ & $\mathrm{~T} 4$ \\
\hline saturados & 36,44 & 34,33 & 38,63 & 35,45 \\
mono-insaturados & 41,54 & 38,35 & 38,59 & 39,93 \\
poli-insaturados & 22,02 & 27,32 & 22,78 & 24,62 \\
\hline
\end{tabular}

Tabla 6. Valores obtenidos para los ácidos grasos saturados en carne de cerdos $(\mathrm{mg} / \mathrm{g})$.

\begin{tabular}{ccccc}
\hline ácido & $\mathrm{T} 1$ & $\mathrm{~T} 2$ & $\mathrm{~T} 3$ & $\mathrm{~T} 4$ \\
\hline$(14: 0)$ & $2,53(0,41)$ & $2,49(0,22)$ & $2,15(0,31)$ & $2,65(0,27)$ \\
$(15: 0)$ & $0,75(0,15)$ & $0,43(0,06)$ & $0,50(0,07)$ & $0,40(0,05)$ \\
$(16: 0)$ & $47,62(7,95)$ & $43,42(3,55)$ & $39,48(4,1)$ & $46,01(4,64)$ \\
$(17: 0)$ & $1,83(0,52)$ & $1,60(0,21)$ & $1,71(0,3)$ & $1,57(0,2)$ \\
$(18: 0)$ & $21,40(2,88)$ & $18,88(1,86)$ & $18,31(1,8)$ & $20,74(2,24)$ \\
\hline
\end{tabular}

Valores expresados en promedio y (entre paréntesis) error estándar.

No se registraron diferencias significativas entre tratamientos.

Tabla 7. Valores obtenidos para los ácidos grasos mono-insaturados en cerdos $(\mathrm{mg} / \mathrm{g})$.

\begin{tabular}{ccccc}
\hline ácido & $\mathrm{T} 1$ & $\mathrm{~T} 2$ & $\mathrm{~T} 3$ & $\mathrm{~T} 4$ \\
\hline $16: 1(9)$ & $6,22(0,97)$ & $6,57(0,5)$ & $5,20(0,59)$ & $7,03(0,64)$ \\
$17: 1(10)$ & $0,48(0,05)$ & $0,770(0,08)$ & $0,78(0,17)$ & $0,64(0,18)$ \\
trans 18:1(11) & $0,84(0,21)$ & $0,45(0,05)$ & $0,63(0,11)$ & $0,49(0,07)$ \\
$18: 1(9)$ & $76,65(14,53)$ & $67,29(6,61)$ & $57,56(6,36)$ & $73,05(8,47)$ \\
\hline
\end{tabular}

Valores expresados en promedio y (entre paréntesis) error estándar. No se registraron diferencias significativas entre tratamientos.

Tabla 8. Concentraciones de ácidos grasos poli-insaturados en cerdos

\begin{tabular}{ccccc}
\hline ácido & $\mathrm{T} 1$ & $\mathrm{~T} 2$ & $\mathrm{~T} 3$ & $\mathrm{~T} 4$ \\
\hline $18: 2(9,12)$ & $29,83(4,46)$ & $36,02(3,6)$ & $28,72(3,26)$ & $32,08(3,4)$ \\
$18: 3(\mathrm{n}-3)$ & $3,73(0,7)$ & $4,52(0,45)$ & $3,19(0,6)$ & $4,68(0,7)$ \\
$20: 2(11,14)$ & $1,17(0,23)$ & $1,23(0,15)$ & $0,96(0,12)$ & $1,04(0,13)$ \\
$20: 3(11,14,17)$ & $4,53(0,46)$ & $3,67(0,49)$ & $4,0(0,63)$ & $3,69(0,53)$ \\
$22: 2(\mathrm{n}-6)$ & $2,88(0,67)$ & $3,49(0,56)$ & $3,47(0,56)$ & $3,60(0,43)$ \\
$22: 6(\mathrm{n}-3)$ & $7,84(2,14)$ & $12,1(1,71)$ & $9,1(2,26)$ & $13,9(1,59)$ \\
\hline
\end{tabular}

Valores expresados en promedio y (entre paréntesis) error estándar. No se registraron diferencias significativas entre tratamientos. 
molido, expeller de soja y suplementadas con aceite de pescado, son presentados en la Tabla 4. De su análisis surge que no se observaron diferencias significativas entre tratamientos.

En la Tabla 5 se muestran las proporciones de ácidos grasos saturados e insaturados obtenidos en los cerdos alimentados con las dietas T1 a T4. Se advierte que los porcentajes de los ácidos grasos mono-insaturados fueron ligeramente superiores en todos los tratamientos, en tanto que los poli-insaturados fueron proporcionalmente menores a los demás.

Las concentraciones de ácidos grasos saturados detectados para cada tratamiento, se describen en la Tabla 6 , donde puede advertirse que las diferencias entre las distintas dietas fueron no significativas. El ácido araquídico (20:0) solo se registró en cuatro unidades experimentales, con un promedio de $0,45 \mathrm{mg} / \mathrm{g}$ de carne.

Los contenidos de los ácidos grasos mono-insaturados registrados para cada tratamiento se describen en la Tabla 7. Los promedios no registraron diferencias significativas entre tratamientos. El ácido nervónico 24:1(15), solo se registró en T1 y T3, en solo dos unidades experimentales, con promedios de 0,68 y $0,62 \mathrm{mg} / \mathrm{g}$ de carne, respectivamente.

Los valores de los ácidos grasos poli-insaturados registrados para cada tratamiento se describen en la Tabla 8. Los promedios no registraron diferencias significativas entre tratamientos. Los ácidos grasos 18:2 (9c, 11 ), $18: 2(10,12), 20: 3(8,11,14)$ y $22: 1$ (9) solo fueron evaluados en algunas unidades experimentales.

En trabajos similares se mencionan aumentos en los niveles de ácidos grasos $\omega 3^{11,12}$, especialmente los de cadena larga como el docosahexaenoico y eicosapentaenoico cuyo precursor es el ácido linolénico. Se afirma que la conversión hepática de estos ácidos a partir del ácido linolénico no es muy eficiente en los mamíferos, por lo cual el aumento de los mismos estaría directamente relacionado a su incorporación en la dieta ${ }^{12}$. La discordancia relacionada con los ácidos $\omega 3$ quizás podría deberse a la diferencia en la duración del ensayo.

En conclusión, los resultados obtenidos permiten inferir que la inclusión de aceite de pescado en la dieta de cerdos no produce modificación en su perfil de ácidos grasos. Esto permitiría realizar suplementaciones lipídicas estratégicas a fin de lograr mejores rendimientos productivos, sin alterar significativamente las propiedades organolépticas y nutricionales de la carne porcina. La obtención de alimentos con propiedades beneficiosas para la salud humana implica un desafío creciente para los diversos sectores productivos, en tanto que el manejo nutricional estratégico constituye una herramienta válida para alcanzar dichos objetivos.

\section{REFERENCIAS}

1. Abeledo C, Santana I, Pérez I, Brache F. 2004. Rasgos de comportamiento y canal de cerdos criollo y cc21 alimentados con palmiche como única fuente de ener- gía. Rev Comput Prod Porc www.iip.co.cu/RCPP/171/ RCPP17.1.pdf.

2. Acosta Sosa MA. 2006. Producción porcina en el NEA. Memorias del $1^{\circ}$ Congreso del NEA de Producción y Carne Porcina, Resistencia (Chaco, Argentina).

3. Belmar-Caso R. 1998. Recursos no convencionales en la alimentación de animales no rumiantes. Public Depto Nutr Anim FMVZ, Univ Autón Yucatán, México, 10 p.

4. Campabadal CM. 2003. Ingredientes utilizados en la alimentación de los cerdos, Asociación Americana de Soya (ASA, México), http://www.soyamex.com.mx.

5. Castaldo DJ. 1998. Focus on feed grade fat. Part 2: fat quality parameters. On line: http://foodtab.com/fat2.hml.

6. Cera KR, Mahan DC, Reinharth GA. 1989. Apparent fat digestibilities and performance responses of post weaning swine fed diets supplemented with coconut oil, corn or tallow. J Anim Sci 67: 2040-2047.

7. Eggert JM, Farrand EJ, Mills SE, Schinckel AP, Forrest JC, Grant AL, Watkins BA. 1998. Effects of supplementing with soybean oil and finishing with beef tallow on pork quality and carcass composition. Swine Day Report 58: $1-8$.

8. FAO. 2004. Perspectivas alimentarias. On line: http:// www.fao.org.

9. Figueroa V. 1997. Integración de la caña de azúcar con el reciclaje de desperdicios, subproductos y derivados para la producción porcina sostenible. Public FAO (Roma), p. 207-225.

10. Hurtado E, González Araujo C. 2002. Aspectos generales y situación actual del cerdo criollo en Venezuela. On line: http://www.portalveterinaria.com.

11. Khiaosa-ard $\mathbf{R}$, Chungsiriwat $\mathbf{P}$, Chommanart $\mathbf{N}$, Kreuzer M, Jaturasitha S. 2011. Enrichment with $n-3$ fatty acid by tuna oil feeding of pigs: changes in composition and properties of bacon and different sausages as affected by the supplementation period. Can J Anim Sci 91: 87-95.

12. Meers SA, Dove CR, Azain MJ. 2006. The effect of dietary omega-3 fatty acids on growth and fatty acid composition of adipose tissue in grower/finisher pigs. On line: http://www.ads.uga.edu/documents.pdf.

13. Moran LA, Scrimgeour KG, Horton HR, Ochs RS, Rawn JD. 1994. Biochemistry, Prentice Hall, New Jersey (USA), p. 475.

14. Muñoz R. 1999. Mercado de carne porcina. Resúmenes de charlas técnicas y conferencias "Fericerdo 99", INTA Marcos Juárez (Córdoba, Argentina), 99 p. .

15. National Research Council-NRC. 1998. Canadian Tables of Feed Composition, National Academy Press, Washington.

16. National Research Council-NRC. 1998. Nutrient requirements of swine, 10th ed., National Academy Press, Washington, $614 \mathrm{p}$.

17. Pochon DO, Navamuel JM, Koslowski HA, Picot JA, Balbuena O. 2007. Estimación de la aceptabilidad de una dieta con sustitución parcial de maíz por mandioca para cerdos en crecimiento. Rev Vet 18: 106-110. 
18. Preston TR, Murgueitio E. 1992. Strategy for sustainable livestock production in the tropics, Ed. Condrit, Cali (Colombia), $89 \mathrm{p}$.

19. Savón L, Scull I. 2001. Valor potencial de alimentos fibrosos tropicales para especies monogástricas. Anales VI Encuentro Regional de Nutrición y Alimentación de Animales Monogástricos, La Habana (Cuba) p. 33.
20. Solsona BS. 2004. Utilización de grasas y subproductos lipidicos en animales. http://www.riosa.com/uploads/images/WBECSNdHmACapgRBF3Txcg/Riosa.pdf.

21. Wiseman J. 1997. Assigning energy values to ingredients for pigs. Proc Feed Ingred, http://www.sian.info.ve/porcinos/publicaciones/producerdos/articulo6.htm.

\section{Asociación Cooperadora de la Facultad de Ciencias Veterinarias Universidad Nacional del Nordeste}

Personería Jurídica No 647/92 y 912/00

Sargento Cabral 2139

3400 Corrientes

Argentina

La Asociación Cooperadora de la Facultad de Ciencias Veterinarias de la UNNE fue constituida el 10 de diciembre del año 1991 como entidad de bien público, con el objeto de promover y coadyuvar las actividades científicas, educativas y culturales relacionadas con las ciencias veterinarias. En tal sentido, implementa acciones para colaborar con la enseñanza, extensión, actualización y difusión científica que realiza dicha casa de estudios.

\section{Beneficios que brinda a sus asociados:}

- Fotocopias con descuentos especiales del $20 \%$ en la Fotocopiadora Copias.com que funciona dentro del predio de la Facultad de Ciencias Veterinarias.

- Descuentos del 10\% para la adquisición de libros de la Editorial Inter-Médica.

- Descuentos especiales en otros rubros.

Sea solidario, asóciese en beneficio de toda la comunidad universitaria

Tel. 03783-425753 interno 186• Página Web www.vet.unne.edu.ar•E-mail: cooperadora@vet.unne.edu.ar 